\title{
Do Patients with Implants Experience Strong Sensations That Lead to Early Termination of MRI Examinations?
}

\author{
J. Steinbacher (D, M. R. McCoy, F. Klausner, W. Wallner, A. Oellerer, and L. Machegger \\ Division of Neuroradiology, Department of Radiology, Paracelsus Medical University Salzburg, Austria Ignaz-Harrer-Strasse 79, \\ A-5020 Salzburg, Austria \\ Correspondence should be addressed to J. Steinbacher; j.steinbacher@salk.at
}

Received 12 March 2019; Accepted 19 May 2019; Published 1 July 2019

Academic Editor: Michele Benedetti

Copyright (c) 2019 J. Steinbacher et al. This is an open access article distributed under the Creative Commons Attribution License, which permits unrestricted use, distribution, and reproduction in any medium, provided the original work is properly cited.

Purpose. Many patients with metallic implants are aware of the potential dangers in an MRI environment. Some implants cause sensations perceptible by the patient during an MRI scan. We wanted to find out if patients having an implant abort MRI scans more often than patients without implants. Furthermore, we wanted to know if the number of implants of a patient has an influence on the number of aborted MRI scans. As we use 3T MRI scanners from different manufacturers we wanted to see the influence of the "ScanWise" software option where scan parameters are adapted automatically to the maximal allowed limits of implant values. This publication should help to reduce possible anxiety in patients with implants. Materials and Methods. From May $15^{\text {th }} 2017$ until July $17^{\text {th }} 2018$ the implant type of patients was recorded. We looked how often aborted MRI scans coincided with the occurrence of implants and the MRI scanner type used. Results. 4088 examinations were collected for the analysis. No influence of implants on aborted MRI scans was registered. No influence of the number of implants on aborted MRI scans was registered. No influence of the use of "ScanWise" software on aborted MRI scans was registered when different scanners were compared. Conclusion. "MR Safe" or "MR Conditional" labelled implants did not have an impact on the MRI examinations. "ScanWise" software does not affect how MRI examinations are tolerated.

\section{Introduction}

The fear of MRI in patients with metallic implant is a growing public health issue. On one hand, the number of MRI examinations increases, and on the other hand, the number of patients with implants increases too. Some patients with implants wonder if they are allowed to enter the MRI environment with their implant at all. Warning and prohibitive signs are ubiquitous when entering the locker rooms. This leads to a further increase in tension in some patients. See Figure 1.

In addition to possible psychological effects of patients with metal implants (or foreign bodies) there are known possible interactions of the implant with the static magnetic field, the radiofrequency field, and the gradient field of the scanner during MRI examinations [1]. This is well known as different kind of artefacts can be found on MRI pictures. An artifact resulting from a small metal foreign body is depicted on Figure 2.
Implants or foreign bodies made of $\mathrm{Fe}, \mathrm{Co}$, and $\mathrm{Ni}$ endure an attractive force and torque by the static magnetic field $\mathrm{B}_{0}$. Many cases with a partly dramatic outcome can be found in literature. The danger of ferromagnetic aneurysm clips [2] or risks of ferromagnetic foreign bodies to the surrounding tissue are also well known $[3,4]$.

The $B_{1}$ field, also known as high frequency field, can cause current induced burns. Various cases can be found in the literature [5]. Metal acts as an antenna in the $\mathrm{B}_{1}$ field. Dempsey et al. [6] found a temperature rise of $0.6^{\circ} \mathrm{C}$ for direct electromagnetic induction in a conductive loop, a rise of $61.1^{\circ} \mathrm{C}$ for induction in a resonant conducting loop, and a rise of $63.5^{\circ} \mathrm{C}$ for resonant coupling with a wire (the antenna effect). Metal implants are not only dangerous if they have the so-called critical wavelength, which is approximately $12 \mathrm{~cm}$ to $13 \mathrm{~cm}$ for a $3 \mathrm{~T}$ scanner [1]. It depends as well on the tissue. Also smaller implants can cause burns [7]. Additionally tattoos made of specific coloring agents can induce burns [8]. 


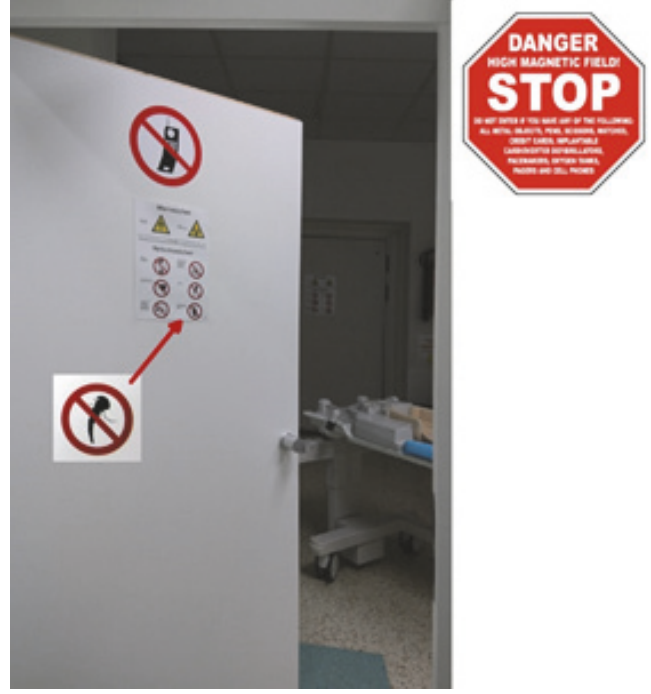

FIGURE 1: Prohibitive signs on MRI doors cause anxiety in some patients. The sign: "entering with implants prohibited" is shown enlarged. "Stop Do Not Enter If You Have Any Of The Following: All Metal Objects..." is a common message at MRI sites.

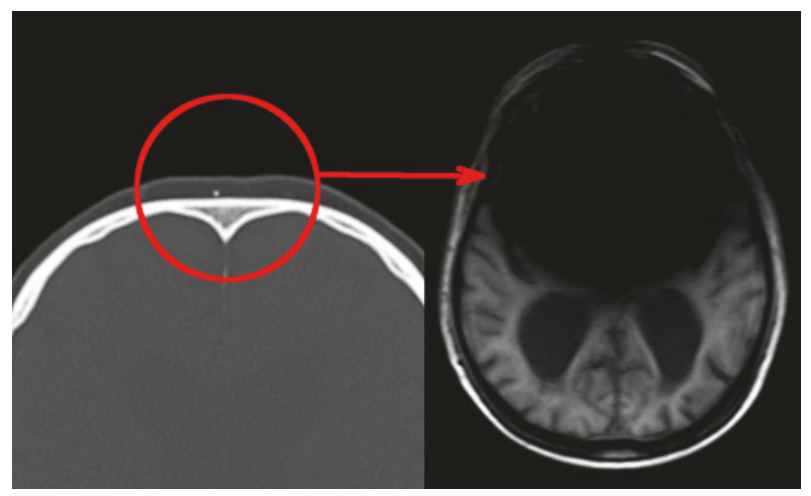

FIGURE 2: Interactions of an MRI scanner and a small metal foreign body. The size of the foreign body is only visible on the CT scan (bone window) on the left side. In comparison the resulting artefact on axial T1. The susceptibility of this small piece of metal disturbs the homogeneity of the $\mathrm{B}_{0}$ field so much that the associated artifact makes up about $50 \%$ of the shown brain.

Safety concerns for the time varying magnetic field gradients $\mathrm{B}_{\mathrm{G}}$ are biological effects: peripheral nerve stimulation, muscle stimulation, and acoustic noise [1,9]. Time varying magnetic field gradients $(\mathrm{dB} / \mathrm{dt})$ can interact with implants. The interaction may result in induced currents, device heating, and vibration of the device. Summing up, the different interactions between MRI and metallic implants are so strong that even MRI-induced stent dislodgment may be possible [10].

As a result, metallic implants were tested for their behavior in MRI environment. The implants are classified into MR Safe, MR Conditional, and MR Unsafe $[1,11]$.. MR Safe implants can be scanned in any scanner. With MR Conditional devices specific modifications, e.g., SAR reduction of the sequence, length of MRI examination, or the use of a transmit-receive coil, are sometimes needed. MR Conditional implants can be scanned in a $1.5 \mathrm{~T}$ or $3 \mathrm{~T}$ depending on the conditions and MR Unsafe implants must not be scanned. Foreign bodies in MRI are a major problem because they cannot be tested in advance.

Over the last couple of years patients reported to our team about sensations after the MRI examination. In many cases, they reported on heat, nerve, and muscle stimulation. As implants made of metal strongly interact with the scanner, we asked ourselves whether patients with implants perceive these sensations more frequently than patients without implants.

\section{Materials and Methods}

From May $15^{\text {th }} 2017$ until July $17^{\text {th }} 2018$ all MRI examinations were collected for analysis.

There were 4864 examinations on both scanners. The patients had either no implants or implants labeled as "MR Safe" or "MR Conditional." This data set was collected retrospectively via analysis of the informed consent documentation (retrospective epidemiologic observer study). All patients gave informed consent for their data to be collected and processed. An approval by an ethics committee was not necessary.

Most patients were scanned via a 3Tesla Philips Achieva Scanner (4361 patients) with "dStream 32ch Head coil," "dStream HeadNeckSpine coil," "dStream T/R Head coil," or "dStream TotalSpine coil." The Philips "ScanWise Implant" software was used for examination on this scanner. After input of limits for parameters as, e.g., spatial magnetic gradient, SAR, and dB/dt by the MRI personal "ScanWise" adjusts scan parameters like TE and TR depending on the limits of an MR Conditional implant to reduce SAR or peripheral nerve stimulation user independent.

Furthermore 503 patients were scanned on a Siemens Magnetom Prisma scanner with either "Head/Neck 64 3T Tim Coil," "Head/Neck 20 3T Tim Coil," or "Spine 32 3T Tim Coil."

The "Magresource MRI SAFETY DATABASE," which is a website where information about implants can be found or experts can be asked about the behavior of not listed implants, was used for MRI safety information of the implants. Patients with unsafe implants were rejected and were not eligible.

In order to obtain data about the type of implant of the patients, MRI radiology technologists interviewed the patients previous of the scans as means of safety screening and documented the findings. We looked at the informed consent documentation and compared the frequency of aborted MRI scans with the occurrence of implants.

When patients talk to radiology technologists, some patients report sensations like noise disturbance and claustrophobia after the examination. It is complex to quantify this information. We used the early termination of the examination by the patient as the main criteria for an MRI scan which was not tolerated by the patient. Criteria for early scan termination were ringing the emergency bell, 
TABLE 1: Detailed information on frequency of implants collected in time span. MR1 means scanner with "ScanWise" option, MR2 means scanner without this software.

\begin{tabular}{|c|c|c|c|c|c|c|c|}
\hline implants & MR 1 & MR 2 & total & additional information & MR1 & MR 2 & total \\
\hline stent & 151 & 7 & 158 & metal foreign bodies & 13 & 0 & 13 \\
\hline coronary stent & 48 & 6 & 54 & surgical titanium mesh & 1 & 0 & 1 \\
\hline heart valve & 27 & 3 & 30 & temporomandibular joint & 0 & 1 & 1 \\
\hline orthopedic implant: screws, plates, nails & 364 & 32 & 396 & lens implant & 2 & 0 & 2 \\
\hline hip joint & 179 & 11 & 190 & tissue marker & 1 & 0 & 1 \\
\hline knee joint & 156 & 10 & 166 & PFO occluder & 3 & 1 & 4 \\
\hline cervical cage & 29 & 2 & 31 & piercing & 23 & 5 & 28 \\
\hline spinal stabilization & 108 & 4 & 112 & dental implant & 296 & 24 & 320 \\
\hline VNS (vagus nerve stimulator) & 5 & 0 & 5 & braces & 64 & 9 & 73 \\
\hline pacemaker & 4 & 0 & 4 & pessary & 1 & 0 & 1 \\
\hline drug infusion system & 3 & 0 & 3 & shoulder joint replacement prostheses & 2 & 0 & 2 \\
\hline cerclage & 27 & 1 & 28 & intrauterine device (IUD) $\mathrm{Cu}$ & 30 & 3 & 33 \\
\hline breast implants & 6 & 0 & 6 & intrauterine device (IUD) $\mathrm{Au}$ & 1 & 0 & 1 \\
\hline gastric band & 6 & 0 & 6 & WEB aneurysm embolization device & 1 & 0 & 1 \\
\hline shunt & 49 & 0 & 49 & CLIP Piston & 2 & 0 & 2 \\
\hline clips & 81 & 5 & 86 & stent esophagus & 1 & 0 & 1 \\
\hline coils & 130 & 0 & 130 & vascular graft & 1 & 0 & 1 \\
\hline port a cath & 37 & 1 & 38 & $\mathrm{BPH}$ treatment Urolift & 2 & 0 & 2 \\
\hline ureteral stent & 1 & 0 & 1 & brachytherapy seeds & 3 & 0 & 3 \\
\hline ankle joint & 2 & 0 & 2 & loop recorder & 2 & 0 & 2 \\
\hline subdural electrodes & 2 & 0 & 2 & & & & \\
\hline
\end{tabular}

discontinuation of the scan, and that the patient could not be convinced to continue the exam.

Statistical analysis was conducted by using Bayestheorem, which describes the probability of an event based on prior knowledge of conditions that might be related to the event. Contingency tables were created, and Pearson's chisquared test $\left(\chi^{2}\right)$ was applied to the data sets to evaluate how likely it is that any observed difference between the sets arose by chance. We used SPSS software for statistical analysis.

\section{Results}

From May $15^{\text {th }} 2017$ until July $17^{\text {th }} 20184864$ MRI were done. The mean age of patients was $59+/-19$ years (mean $+/-\mathrm{SD}$ ). The minimum age was 1 year and the maximum age was 98 years. $51.6 \%$ were female. The mean age of female patients was $58+/-20$ years (mean +/-SD), minimum age 1 year, and maximum age 98 years. The mean age of male patients was $59+/-18$ years (mean +/- SD), minimum age 4 years, and maximum age 95 years. As patients under anesthesia could not stop the scan, those were excluded from this work, as well as patients with psychiatric comorbidities such as anxiety disorders or phobias. In patients who received several examinations, only the first examination was considered. That leads to 4088 MRI examinations which are included in the statistical analysis. 1625 patients (equal 40\%) had implants when they were scanned. Table 1 displays detailed information on the frequency of implants. Table 2 shows information on the number of stopped scans and the total number of scans with and without implants.
Question 1: Do patients with implants abort MRI scans more often than patients with no implants?

To answer question 1 we looked at all scans included in the time span. From 4088 scans 1625 were with implants and 2463 without implants. Patients with implants aborted in 9 cases, patients having no implants aborted in 20 cases. We used the odds ratio, which is the ratio of "chances" for "abort or no abort" between the implant and nonimplant group. The odds ratio in this case is 0.68 which is lower than 1 . This indicates that the "chances" in the implant group for a termination of the exam is not greater, compared to the other group. The number of degrees of freedom is 1 . The upper-tail critical values of chi-square distribution table (Table 3 ) give a critical value of 3.84 at $95 \%$ significance level. As $\chi^{2}$ is 0.93 , there is no influence on more aborted scans from patients with implants than from patients without implants.

Question 2: Do patients with more than one implant abort MRI scans more often than patients with a single implant?

To answer question 2 we compared patients having one or two implants. We looked at all scans from patients having one or two implants included over the previously mentioned time span. See Table 4.1285 scans were from patients having one implant and 238 scans from patients with two implants. Patients having one implant aborted in 6 cases; patients having two implants aborted in 2 cases. We use the odds ratio, which is the ratio of "chances" for "abort or no abort" between the one implant and two-implant group. The odds ratio in this case is 0.55 which is lower than 1 . This indicates that the "chances" in the two-implant group for a termination of the exam is not greater, compared to the other group. The number 
TABLE 2: Detailed information on stopped scans with and without implants, and total number of scans with and without implants.

\begin{tabular}{lccc}
\hline information regarding scans & number & number & number \\
\hline stopped scans without implant & 27 & 2 & 29 \\
stopped scans with implants & 8 & 1 & 9 \\
total number of scans & 3817 & 271 & 4088 \\
total number of scans without implant & 2300 & 163 & 2463 \\
total number of scans with implant & 1517 & 108 & 1625 \\
\hline
\end{tabular}

TABLE 3: Contingency table aborted and finished scans vs. implant yes / no.

\begin{tabular}{lccc}
\hline & scan aborted & scan finished & total \\
\hline implant & 9 & 1616 & 1625 \\
no implant & 20 & 2443 & 2463 \\
total & 29 & 4059 & 4088 \\
\hline
\end{tabular}

TABLE 4: Distribution of MRI scans of patients having multiple implants.

\begin{tabular}{lcc}
\hline No. Implants & No. of scans & No. of stopped scans \\
\hline 1 & 1279 & 6 \\
2 & 236 & 2 \\
3 & 47 & 0 \\
4 & 10 & 1 \\
\hline
\end{tabular}

TABLE 5: Contingency table aborted and finished scans vs. 1 implant / 2 implants.

\begin{tabular}{lccc}
\hline & scan aborted & scan finished & total \\
\hline 1 implant & 6 & 1279 & 1285 \\
2 implants & 2 & 236 & 238 \\
total & 8 & 1515 & 1523 \\
\hline
\end{tabular}

of degrees of freedom is 1 . The upper-tail critical values of chisquare distribution table (Table 5) show a critical value of 3.84 at $95 \%$ significance level. As $\chi^{2}$ is 0.54 there was no influence on more aborted scans from patients with two implants than from patients with just one implant.

To answer question 2 we also compared patients having one implant with patients having three implants. We looked at all scans from patients having one or three implants included over the previously mentioned time span, 1285 scans from patients having one implant and 236 scans from patients having two implants. Patients having one implant aborted in 6 cases, patients having three implants aborted in 2 cases. We used the odds ratio, which is the ratio of "chances" for "abort or no abort" between the one implant and three-implant group. The odds ratio is not defined in this case. A proper statement for patients with three implants cannot be made due to the low number of scans conducted. See Table 6.

Question 3: Do patients with implants that are examined without "ScanWise" software terminate MRI examinations more often than patients examined in a scanner with "ScanWise" software (or a similar product)?
TABLE 6: Contingency table aborted and finished scans vs. 1 implant / 3 implants.

\begin{tabular}{lccc}
\hline & scan aborted & scan finished & total \\
\hline 1 implant & 6 & 1279 & 1285 \\
3 implants & 0 & 47 & 47 \\
total & 6 & 1326 & 1332 \\
\hline
\end{tabular}

TABLe 7: Contingency table aborted and finished scans vs. "ScanWise" yes / no.

\begin{tabular}{lccc}
\hline & scan aborted & scan finished & total \\
\hline "ScanWise" & 8 & 1509 & 1517 \\
no "ScanWise" & 1 & 107 & 108 \\
total & 9 & 1616 & 1625 \\
\hline
\end{tabular}

To answer question 3 we looked at all scans with implants included in the time span. 1517 scans were done on the Philips 3T scanner with "ScanWise" software. 108 scans were done on the Siemens 3T scanner without a similar "ScanWise" option. Patients in the Philips scanner aborted in 8 cases; patients in the Siemens Scanner aborted in 1 case. We use the odds ratio, which is the ratio of "chances" for "abort or no abort" between the "ScanWise" and not "ScanWise" group. The odds ratio in this case is 0.57 which is lower than 1 . This indicates that the "chances" in the "ScanWise" group for a termination of the exam is not greater, compared to the other group. The number of degrees of freedom is 1 . The upper-tail critical values of chi-square distribution table give a critical value of 3.84 at $95 \%$ significance level. As $\chi^{2}$ is 0.29 we found no influence on more aborted scans from patients scanned on a scanner using "ScanWise" software than for patients scanned on a scanner not using "ScanWise" software. See Table 7.

\section{Discussion}

Metallic implants can cause problems apart from artefacts in MRI examinations. Implants made of ferromagnetic metals are dragged into the powerful static magnetic field $\mathrm{B}_{0}$ of the MRI scanners [1]. Torsional forces act on elongated ferromagnetic objects [1]. Radiofrequency HF-fields $B_{1}$ can cause heating of metallic implants [1]. Some of the aforementioned cases led to serious injuries [12,13]. Time-dependent gradient-fields $\mathrm{B}_{\mathrm{G}}$ can cause electrical voltage [14], heating, noise, and vibrations [15] on implants made from metal. Metallic implants cause a major safety problem in MRI examinations. There are well known publications on the 
management of implants in daily routine [11]. However, we are not aware of any fundamental publication in which the frequency of implants or safety-relevant cases in MRI exams has been the subject of research.

All patients have to undergo a prescan check for possible implants. These implants have to be either MR Conditional regarding our MRI in use at the "Christian Doppler Klinik" or MR Safe. Checking for implants previous to the MRI scan has led to higher expenditures over the past years. Therefore we were interested in absolute figures of implants scanned in the neuroradiology at "Christian Doppler Klinik." We found that approximately $40 \%$ of our patients had implanted devices when they were examined.

Over the past years patients reported what they perceived in the scanner to our MRI technologists. So we know that perception of sensations like, pain, noise, heat, and claustrophobia is different for every individual. As an example, we want to mention the case of a patient having a tribal tattoo on the left upper arm. After the scan the patient complained about heat sensation at the position of the tattoo. For him it was very hard to bear, but he was convinced the scan was of such importance that he did not want to terminate the exam prematurely. In contrast to the previous case, a girl stopped the examination immediately after the beginning as she felt a heat sensation at the position of her braces. These cases give a perfect example of how difficult it is to rate sensations during a MRI scan. Abortion of the scan by the patient as a result of different kinds of discomfort was the only clear criteria of our study.

As mentioned above, implants can cause additional sensations during MRI scan that subsequently lead to our first hypothesis: patients having implants would stop MRI examinations significantly more often. Secondly, we thought that the number of implants would have an influence on MRI scans. As we use 3T scanners of different manufacturers, of which one has software to adapt the MRI scans to the implants boundary conditions ("ScanWise by Philips) at our institute, we set hypothesis 3. Patients with implants scanned without "ScanWise" option stop MRI examinations more often. Each of these three hypotheses was refuted.

No patient harm due to side effects of metallic implants, as mentioned above, has been documented.

During the period of our data collection, the further following safety-related events occurred: One patient suffered from an epileptic seizure during the MRI exam. This patient had no implants. One patient suffered from a cardiovascular arrest in the MRI scanner and had to be reanimated. This patient had no implants. There was an incident during contrast agent administration where the patient had to throw up at the moment of contrast agent administration. This patient had an orthopaedic implant, a knee joint, gastric banding, a loop recorder, and a tattoo. The contrast agent allergy was not known at this point. Furthermore two incidents related to contrast agent administration occurred after the examination when the patient had already left the scanner room. In addition we found the following types of implants or combinations of implants from patients who aborted the MRI examination: cerclage only, cerclage and dental implant, dental implant only (two cases), hip joint, orthopaedic implant, stent cranial, coronary stent, and heart valve.

Below we listed specific limitations of this study. A limitation of this work is that we compared two different scanners and not the same scanner with or without the "ScanWise" software option. But we do not see the effect we suspected: less scans stopped with "ScanWise" software. Different levels of tolerance from the patients to stop an examination are a limitation too. Some MRI technologists communicate in a more sensitive way with patients and can persuade patients to continue the examination after ringing the emergency bell. A key point why burns can occur during MRI scans, even without implants, is the lack of heat receptors in soft tissue [16]. This heat induced burns are unlikely to be detected during the examination when the hotspot is distant from sensory tissue that could alarm the patient to immediately terminate the scan. This could probably lead to undetected cases. We would like to pursue this question in a future study.

\section{Conclusion}

All our hypotheses were refuted.

We did not find any significance regarding more prematurely stopped scans from patients with implants than for patients without implants.

We did not find any significance regarding more prematurely aborted scans from patients with two or three implants than from patients with one implant.

We did not find significant more aborted MRI examinations from patients scanned on a unit using "ScanWise" software than for patients examined on a unit without "ScanWise" software.

Even though there are a large number of publications on burns and incidents during MRI examinations, we could now prove that MRI examinations are just as tolerable for patients with MR Conditional implants as for patients without MR Conditional implants.

\section{Data Availability}

Data processed in this work can be found in Tables 1 and 2 .

\section{Conflicts of Interest}

On behalf of all authors, the corresponding author states that there is no conflict of interest.

\section{References}

[1] EN 60601-2-33 Medical electrical equipmentPart 2-33, "Particular requirements for the basic safety and essential performance of magnetic resonance equipment for medical diagnosis," Tech. Rep. IEC 60601-2-33, 2010.

[2] R. P. Klucznik, D. A. Carrier, R. Pyka, and R. W. Haid, "Placement of a ferromagnetic intracerebral aneurysm clip in a magnetic field with a fatal outcome," Radiology, vol. 187, no. 3, pp. 855-856, 1993. 
[3] T. E. S. Omar, U. El-Khani, and J. Nehme, "MRI-induced soft tissue pain: incidental finding of a 15-year-old foreign body," BMJ Case Reports, vol. 2013, Article ID bcr2012008275, 2013.

[4] W. M. Kelly, P. G. Paglen, J. A. Pearson, A. G. San Diego, and M. A. Soloman, "Ferromagnetism of intraocular foreign body causes unilateral blindness after MR study," American Journal of Neuroradiology, vol. 7, pp. 243-245, 1986.

[5] M. V. Knopp, R. Metzner, G. Brix, and G. van Kaick, "Sicherheitsaspekte zur Vermeidung strominduzierter Hautverbrennungen in der MRT," Der Radiologe, vol. 38, no. 9, pp. 759-763, 1998.

[6] M. F. Dempsey, B. Condon, and D. M. Hadley, "Investigation of the factors responsible for burns during MRI," Journal of Magnetic Resonance Imaging, vol. 13, no. 4, pp. 627-631, 2001.

[7] L. J. Kim, V. K. H. Sonntag, J. T. Hott, J. A. Nemeth, J. D. Klopfenstein, and L. Tweardy, "Scalp burns from halo pins following magnetic resonance imaging: Case illustration," Journal of Neurosurgery, vol. 99, no. 1, article 186, 2003.

[8] D. Klitscher, J. Blum, K. F. Kreitner, and P. M. Rommens, "MRT-induzierte Verbrennung bei TätowierungenMRIinduced burns in tattooed patients," Der Unfallchirurg, vol. 108, no. 5, pp. 410-414, 2005.

[9] D. Grainger, Safety Guidelines for Magnetic Resonance Imaging Equipment in Clinical Use, Medicines and Healthcare Products Regulatory Agency, London, UK, 2014.

[10] H. Parthasarathy, O. Saeed, D. Marcuzzi, and A. N. Cheema, "MRI-Induced stent dislodgment soon after left main coronary artery stenting," Circulation: Cardiovascular Interventions, vol. 6, no. 5, pp. e58-e59, 2013.

[11] E. Kanal, A. J. Barkovich, C. Bell et al., "ACR guidance document on MR safe practices: 2013," Journal of Magnetic Resonance Imaging, vol. 37, no. 3, pp. 501-530, 2013.

[12] J. Haik, S. Daniel, A. Tessone, A. Orenstein, and E. Winkler, "MRI induced fourth-degree burn in an extremity, leading to amputation," Burns, vol. 35, no. 2, pp. 294-296, 2009.

[13] E. Mattei, M. Triventi, G. Calcagnini et al., "Complexity of MRI induced heating on metallic leads: experimental measurements of 374 configurations," Biomedical Engineering Online, vol. 7, article 11, 2008.

[14] S. Feng, R. Qiang, W. Kainz, and J. Chen, "A technique to evaluate MRI-induced electric fields at the ends of practical implanted lead," IEEE Transactions on Microwave Theory and Techniques, vol. 63, no. 1, pp. 305-313, 2015.

[15] G. Schaefers, M. Scholten, P. Bartnik, A. Douiri, and W. Görtz, "MR testing of gradient-induced vibrations using an optical contact-free sensor within the switched gradient magnetic field of a 1.5 Tesla MR system," in Proceedings of the International Society for Magnetic Resonance in Medicine (ISMRM), vol. 57, Wiley, 2012.

[16] P. V. Viscuse, M. Khasawneh, and C. Constantinou, "MRiinduced second and third degree burns to hands bilaterally: a case report," Annals of Hematology \& Oncology, vol. 2, no. 7, article 1053, 2015. 


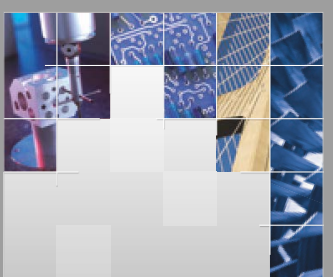

\section{Enfincering}
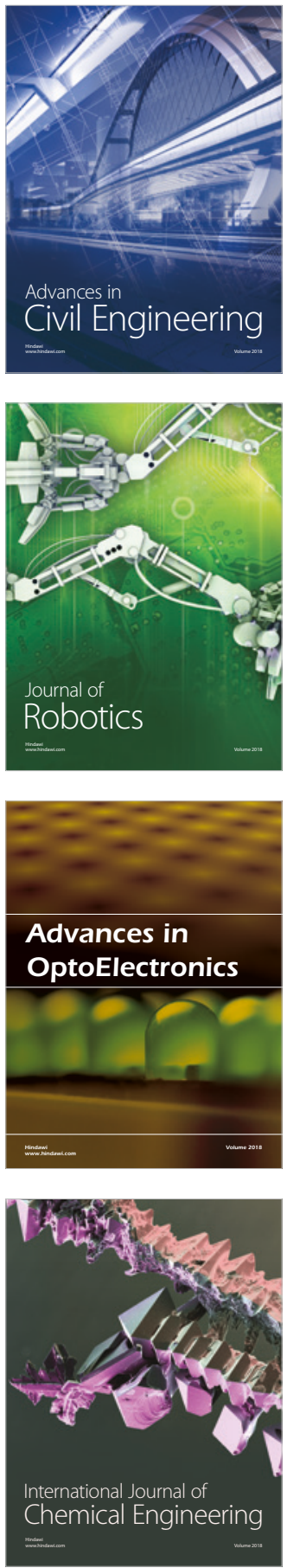

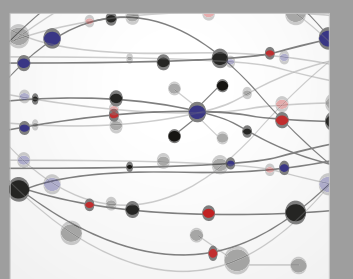

\section{Rotating \\ Machinery}

The Scientific World Journal

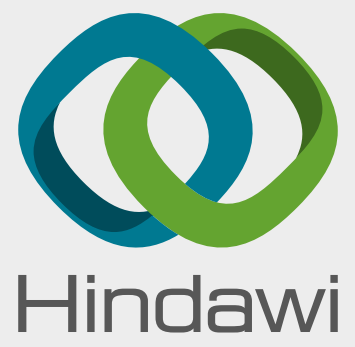

Submit your manuscripts at

www.hindawi.com
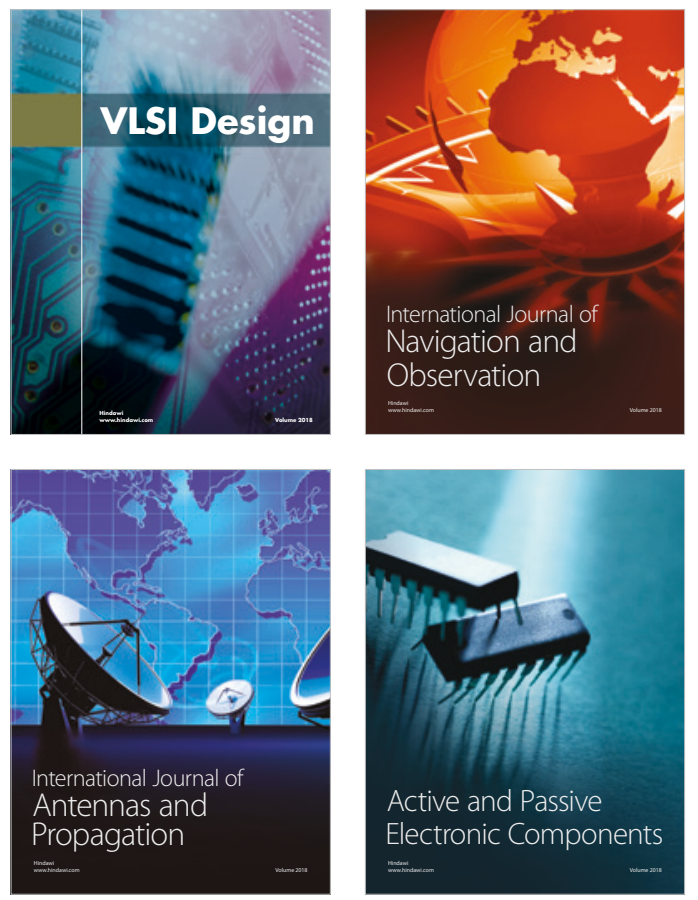
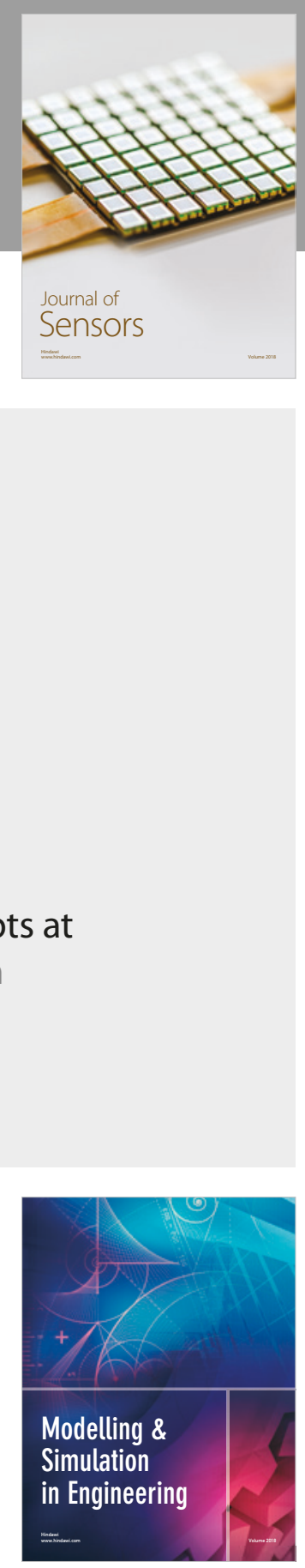

\section{Advances \\ Multimedia}
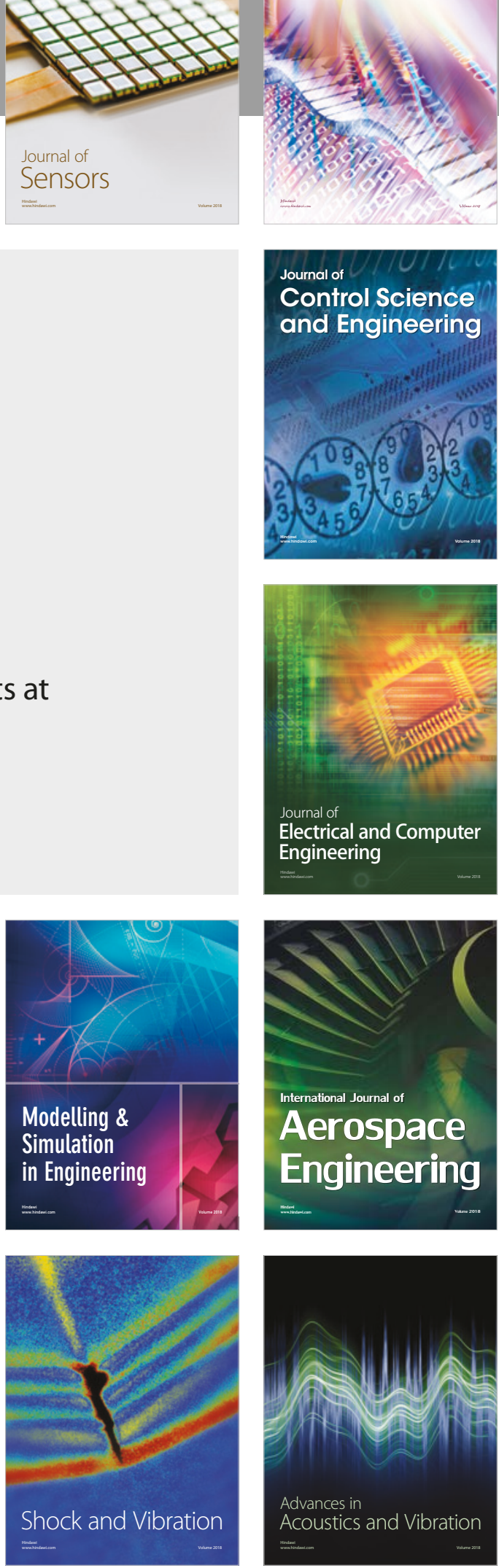